Voix et Images

volxetimages

\title{
Constantes et ruptures en recherche littéraire : le cas d'Études littéraires
}

\section{Jean-Marcel Paquette}

Volume 12, numéro 2 (35), hiver 1987

Jacques Brault

URI : https://id.erudit.org/iderudit/200634ar

DOI : https://doi.org/10.7202/200634ar

Aller au sommaire du numéro

Éditeur(s)

Université du Québec à Montréal

ISSN

0318-9201 (imprimé)

1705-933X (numérique)

Découvrir la revue

Citer cet article

Paquette, J.-M. (1987). Constantes et ruptures en recherche littéraire : le cas d'Études littéraires. Voix et Images, 12(2), 271-277.

https://doi.org/10.7202/200634ar d'utilisation que vous pouvez consulter en ligne.

https://apropos.erudit.org/fr/usagers/politique-dutilisation/ 


\title{
Constantes et ruptures en recherche littéraire: le cas d'Études littéraires
}

\author{
par Jean-Marcel Paquette, Université Laval
}

Voici donc la cadette des trois revues qui forment l'objet de ce colloque: elle est issue de la doyenne des trois universités représentées. Vous assisterez. d'abord à une description analytique du corpus des Etudes littéraires, que je soumettrai ensuite à trois questions: a) qui y écrit? - c'est le domaine des collaborations b) sur quel sujet? - permettant de décrire le contenu c) comment? - qui élucidera la question des méthodes.

Pour des raisons de commodités, j’ai volontairement limité le corpus à ce qu'en dit l'Index publié à l'occasion du quinzième anniversaire de sa première parution, en conséquence aux années 1968 à 1983, soit 46 numéros représentant au total 309 articles. Dans ce dernier compte. j’ai délibérément laissé de côté les "présentations" de numéro (parfois assez. longues). de même que les comptés rendus: et je ne ferai qu'accessoirement appel à une section de la revue placée sous la rubrique "document".

La revue des Études littéraires (et c'est son originalité lorsqu’elle paraît) se présentant comme résolument thématique, c'est par le thème en premier lieu qu'il s'avérera pertinent de l'interroger dans le but de faire un inventaire de ses préoccupations. On doit savoir, lorsque d'aventure on a été mêlé à la direction d'une revue, à quelles nécessités souvent et à quels aléas sont soumises les équipes ayant la charge de préparer les numéros. Mais en l'absence de tout savoir précis sur ces contraintes parfois seulement devinées, voici à quoi se réduit en très gros la mise "en série» de la revue: deux numéros (et 
c'est déjà probablement effet de contrainte) sont constitués par des Actes de colloque, l'un consacré à la poésie contemporaine (1968), l'année de la naissance de la revue; l'autre à la place de la littérature dans la culture d'aujourd'hui (1973). Deux numéros sont consacrés aux relations littéraires entre la France et l'Espagne aux $18^{\mathrm{e}}$ et $19^{\mathrm{e}} \mathrm{s}$. (1969), entre la France et l'Allemagne au $20^{\mathrm{e}} \mathrm{s}$. (1970); trois numéros sont orientés par des époques : «Roman et théâtre au $18^{\mathrm{e}}$ s." (1968), "Le roman médiéval » (1971), "La pensée au 16. $\mathrm{e}$.» (1971) - donc dans les premières années de la revue. Huit numéros sont résolument consacrés au corpus québécois: "Le roman» (1969), "La poésie actuellè " (1972), “L'américanité» (1975), «La Nouvelle-France» (1977), «lXE-13» (1979), "Histoire littéraire du Québec et sémiotique textuelle" (1981), "La consommation littéraire de masse au Québec» (1982) et un numéro sur la littérature québécoise vue par des analystes brésiliens (1983).

Huit numéros, de même, sont consacrés à des thèmes inclassables, dits pour cela "autres": "La paralittérature" (1974), "La littérature négroafricaine" (1974), "La littérature féminine" (1979), "Le récit cinématographique" (1980), "La littérature haïtienne" (1980), "La fiction télévisée" (1981), "La didactique de la littérature dans les collèges classiques du Québec" (1981), enfin, "Le roman 'rose' ou sentimental» (1983).

Neuf numéros, selon ce qui semble être une politique irrégulièrement annuelle de la revue, sont consacrés à des auteurs: Baudelaire (1968). Gide (1969). Alphonse Daudet (1971), Aimé Césaire (1973), Gilbert Langevin (1973). Claude Simon (1976), Lautréamont (1978), Marguerite Yourcenar (1979), Giono (1982). On voit ici la difficulté du classement: Langevin, par exemple, placé sous cette rubrique, pouvait tout aussi bien figurer sous celle du corpus québécois - mais nous reviendrons plus loin sur cette question.

Enfin, quatorze numéros font état de problématiques considérées dans le sens large comme étant "de théorie» se rapportańt à des genres: "Techniques romanesques" (1970), "L'essai" (1972), "La poésie moderne», "Le pamphlet " (1978). "Le théâtre" (1980); à l'inter-disciplinarité: "Critique et didactique" (1970), "La littérature comparée» (1974), "Linguistique et littérature" (1976). "Littérature et philosophie " (1976), "Littérature et musique" (1976); à la diffusion d'univers théorique peu connu: «La théorie littéraire dans le monde hispanique», (1975); à des approches théoriques particulières: «Sémiotique du discours» (1975). «Psychocritique» (1979), «L'énonciation» (1983).

Ces numéros comportent en moyenne de six à sept articles: nettement en dessous de cette moyenne. le ruméro consacré au roman québécois (trois articles), nettement au dessus: seize articles pour les Actes du colloque de 1973 sur la littérature dans la culture d'aujourd'hui. S'approchant d'assez loin de cette dernière abondance, les dix et onze articles des numéros de paralittérature et de littérature féminine. Cette surreprésentation s'expliquant par le fait que ces numéros sont constitués de résultats de projets de recherches. le plus souvent préparés entièrement par des étudiants chargés de recherche de l'université Laval. 


\section{Qui écrit?}

Êtudes littéraires est officiellement la revue littéraire de la Faculté des Lettres de l'Université Laval. Or, un seul numéro ("La pensée au $16^{\mathrm{e}} \mathrm{s} . »$ ) est entièrement et exclusivement dû à des professeurs du Département des littératures. Á l'opposé, sept numéros ne requièrent la collaboration d'aucun professeur de Laval ("Le pamphlet" 78, "La psychocritique" 78, "Le récit télévisé» 81, "La sémiotique du théâtre» 80 , "La littérature de masse au Québec» 82, "Giono" 82 et "Le roman sentimental» 83). Ce dernier, de même que le numéro consacré à la psychocritique, fait une très large place aux étudiants de doctorat du Département, regroupés dans des projets collectifs de récherches.

La revue fait le plus souvent appel à des professeurs d'autres départements de l'Université Laval, surtout lorsqu'il est question d'interdisciplinarité (linguistique, philosophie, musique, anthropologie). Mais la dominante demeure la collaboration non seulement extérieure à l'université mais extérieure au Québec. Six numéros seulement sont conçus sans collaboration extérieure au Québec; de ces six numéros, cinq sont consacrés au corpus québécois et de ces cinq, trois sont des résultats de projets de recherches dirigés du Département des littératures de Laval. La quasi totalité des professeurs de littérature française de ce Département a un jour ou l'autre apporté sa contribution à la revue; ce qui n'est pas le cas des professeurs de littérature québécoise dont la participation est faible: deux seules contributions à la date de 1983. Ce qui explique sans doute que ce soit précisément un numéro consacré au roman québécois qui ait la plus faible moyenne d'articles (3). Il faut aussi compter qu'un numéro de québécois a été entièrement constitué de collaborateurs extérieurs au Québec (Brésil, 1983). On remarque de même peu de collaborations venant des autres secteurs linguistiques du Département des littératures: une seule pour chacun des secteurs d'études anciennes, d'allemand. d'anglais et d'espagnol. Mais, on le voit suffisamment, Études littéraires n'est pas pour autant la revue du Département, sa caractéristique étant la participation extérieure (au Québec ou à l'étranger).

Et c'est ici le lieu de faire état des collaborations "prestigieuses», celles que l'on peut identifier comme des "têtes d'affiche" d'une école ou d'une tendance critique: il y a Starobinski, Zumthor, Ricardou, Mounin. Meschonnic, Kristeva, M.-C. Ropars (en cinéma), ce qui marque une certaine affinité avec les tendances dites "nouvelles" en matière critique. Dans le temps, ce qui est peut-être significatif, ces collaborations prestigieuses se regroupent principalement autour des années 1976-77. Dans la section "document", on publie des inédits de prestige: Senghor, Gide, Reverdy, Benoist-Méchin, Luckacs (un texte alors inédit en français), Césaire. Yourcenar. Purement documentaire, cette publication d'inédits, qui s'apparente à une tendance "historicisante". marque une pointe plutôt au début des années 70 (entre 68 et 73): un seul inédit, celui de Marguerite Yourcenar. étant de 79. Signalons à ce point-ci que parmi les écrivains, un seul parmi les Québécois a participé à la revue; il s'agit de Fernand Ouellette, plus ou moins apparenté à Laval par une charge de cours qu'il y assura durant quelques années. 


\section{Les sujets}

On peut remarquer que deux des sept séries de notre classement, les moins nombreuses (deux numéros pour le comparatisme, trois pour des époques), sont surtout associées aux débuts de la revue (entre 1968 et 1971) - non pas que ces sortes de sujets aient été ensuite négligés, mais quand il s'est agi. plus tard en 1974, par exemple. de traiter de comparatisme, le traitement passait du corpus proprement dit au théorique. De même, alors que le numéro québécois portant sur la Nouvelle-France aurait pu être inventorié comme numéro "d'époque", en 1977 la notion de corpus québécois avait suffisamment évolué pour y faire admettre cette période pouvant se rattacher de façon ambiguë à la fois au corpus français et au corpus québécois. On voit ainsi que certains titres figurant en inventaire sous une rubrique aurait pu parfois figurer sous une autre, n'était le contexte qui invitait à les y joindre. C"est ainsi. encore, que nous avons placé sous la rubrique «numéro d'auteur" le "Langevin" de 1973 qui aurait pu tout aussi bien figurer sous la rubrique "corpus québécois", n'était ce qui semblait alors une politique éditoriale d'un numéro annuel consacré à un auteur. Langevin. d'ailleurs, est le seul auteur québécois représenté dans la série des neuf numéros d'auteur, dont six (y compris Langevin) sont du 20e s. et trois du 19e: Baudelaire et Daudet aux débuts de la revue (1968-71). Lautréamont en 1978 - celui-ci étant un auteurfétiche d'une certaine modernité critique. 11 ne faut pas croire pour autant que la revue, sur ce point, suit d'office une mode: les numéros sur Daudet, Césaire, Simon, Giono marquent un souci évident d'originalité, de même pour Yourcenar qui, en 1979, n'était pas encore l'auteur-à-la-mode qu'elle est devenue depuis. C'est ainsi que se crée une certaine continuité, du moins une cohérence certaine dans la complémentarité entre numéros d'époque (où sont représentés le Moyen Âge, le $16^{\mathrm{c}}$ et le $18^{\mathrm{e}} \mathrm{s}$.) et les numéros d'auteur (exclusivement des $19^{\circ}$ et $20^{\mathrm{e}} \mathrm{s}$.) - un grand trou remarqué: le $17^{\mathrm{e}}$ siècle, qui a pourtant servi de corpus aux textes fondateurs de toutes les écoles de «nouvelles critiques"; que l'on songe au Racine de Mauron, au Racine de Barthes. au Racine de Goldmann.

L'originalité, dont nous venons de faire état, est à son comble dans la série des numéros consacrés à des sujets que nous avons dits "autres»-il y a originalité, en effet, pour une revue littéraire et dans le contexte québécois de 1974 à 1983, à consacrer quelques numéros à la paralittérature, à l'écriture féminine, aux littératures d'Afrique et d'Haïti, au récit filmique ou télévisé. Mais, paradoxalement, c'est sur ces points que la politique éditoriale de la revue semble céder le plus aimablement aux contraintes de la mode du moment: 74-83 sont, en effet. les années où l'on voit s'affirmer avec le plus d'ampleur tant en Europe que sur notre continent, les recherches d'envergure sur le statut de la paralittérature, sur celui de la femme à travers l'écriture, sur celui des littératures «noires", africaine ou antillaise, enfin c'est l'époque où l'enseignement des lettres intègre, de façon diverse selon les lieux, celui de l'audio-visuel (cinéma/télévision): tout l'éventail des modes du temps se trouve ici respectueusement représenté.

Entre les mêmes années, ces modes sont également transposées dans la production de la série du corpus québécois: le numéro «IXE-13», celui sur la 
consommation littéraire de masse ressortissent à la paralittérature; celui sur "l'américanité" répond, par identification, à l'affirmation des littératures noires. Une originalité absolue cependant: celle du numéro «littérature du Québec» entièrement conçu par des professeurs du Brésil - mais on a vu que la contribution lavalienne des professeurs de québécois était, dans l'ensemble. numériquement faible. Pour le reste, la série québécoise, à ses débuts (69-72), procède par traitement traditionnel des genres périodisés: le roman 1945-1960 (1969), la poésie actuelle (1972).

Une autre originalité: le numéro de 1981 où à l'inévitable "historicisation " du corpus québécois s'ajoute la non moins inévitable "sémiotique" -le numéro porte alors le curieux titre de "Sémiotique textuelle et histoire littéraire du Québec's. À une collaboration près, ce numéro de six articles de fond est composé de contributions extérieures à Laval.

C'est dans sa série peut-être improprement désignée comme de «théorie» que l'innovation semble la plus grande: avec sa sous-série des "littérature et linguistique", "littérature et philosophie", "littérature et musique» s'affirme moins une dépendance à la mode de la théorie qu'une réelle ouverture sur des champs qui n’avaient. ici du moins, jamais encore été explorés. C'est d'une pareille ouverture que procèdent les numéros sur la théorie dans le monde hispanique et la théorie de la littérature comparée.

À l'exception de la sociocritique, toutes les approches «nouvelles" du texte littéraire sont représentées: poétique et narratologie ( 3 numéros). sémiotique ( 2 numéros), psychocritique ( 1 numéro). sans compter. en début de série, ce numéro de 1970 où toutes ces nouvelles critiques sont d'un seul coup mises au service d'une pédagogie renouvelée de l'enseignement littéraire. $\mathrm{Cu}$ rieusement, c'est dans ce numéro que se trouve le seul article de la série consacré à la théorie de la sociologie littéraire: il est dû, non moins curieusement, à Jean Starobinski.

Dans la théorie des genres, abordée de façon non traditionnelle, on innove également en consacrant deux numéros à des genres à vrai dire peu théorisés: le pamphlet et l'essai. Ce dernier numéro est même une innovation "mondiale» dans le petit monde du moins des études littéraires - cette théorisation de l'essai est même devenue depuis lors, en quelque sorte, une spécialité québécoise. Même la célèbre revue polonaise consacrée aux genres littéraires n’avait jusqu’à ce jour ouvert ses pages au genre "mineur» de l'essai.

\section{Comment}

Nous voici en présence. c'est le moins qu'on puisse dire. d'un éventail assez complet des principales préoccupations qui, en études littéraires, dans les mêmes années, agitaient et Paris et Berkeley. Essayons maintenant d'examiner et de décrire de plus près comment se réalise cette diversité. Pour ce faire, j’ai retenu, au hasard, un numéro de chacune des quatre grandes séries du début, soit: théorie, corpus québécois, auteurs et "autres". 
Soit le numéro «Littérature et philosophie»:

-- Un premier article, de facture traditionnelle, interroge l'attitude des philosophes devant l'acte littéraire: c'est une présentation, sous forme de dissertation, de la nature proprement historique des liens entre littérature et philosophie, depuis les Grecs jusqu'aux philosophes modernes: L'auteur, un sémioticien en philosophie, utilise ici une méthode justifiée par le fait qu’il informe un public (celui des littéraires) considérés comme non initiés au corpus des philosophes. Méthode descriptive, donc, à incidence historique.

- L'article suivant en dit long par sun titre: "Théorie du langage, théorie pratique: Humboldt et Saussure selon Chomsky". L'interrogation se fait ici par le biais d'une épistémologie de la linguistique moderne et des conséquences idéologiques de sa transplantation dans le monde universitaire américain. Cet article, participant à une polémique alórs en cours sur la grammaire générative, marque un des temps forts de cette lutte. L'article est depuis souvent cité. Il est de Meschonnic.

- Vient une étude où intervient de façon fort pertinente la phénoménologie et la sémiotique greimassienne, donnant lieu à une problématique ouverte sur la théorie des récits.

- Suit une étude hautement critique sur les utilisations inconscientes d'Aristote dans les théories modernes du discours.

- Enfin, une étude sur la conception des rapports entre philosophie et pensée chez Heidegger.

Par la voie d'une thématique associant philosophie et littérature, nous voyons que se trouvent soulevées certaines grandes questions qui agitent pour lors l'activité théorique du moment: Chomsky, Greimas - plus près de ce monde linguistique qui hante les études littéraires - ne restent pas en dehors de la préoccupation des philosophes, qui les interrogent sous une facette résolument nouvelle pour les littéraires. Le numéro, sur cinq articles, comporte une collaboration de Laval, une européenne et trois d'autres universités du Québec.

Du corpus québécois, le numéro «IXE-13"; dix contributions, toutes de Laval, dont une extra-départementale, et cinq (la moitié) d'étudiantschercheurs engagés dans un projet collectif. Ce numéro comporte:

— une étude interdisciplinaire cinéma/texte selon la méthode la plus orthodoxe de la transformation des récits inspirée de Lévi-Strauss;

- une étude d'institution littéraire sur les données économiques de la diffusion du roman policier;

- une étude psychocritique du corpus d'IXE-13;

- une étude de classification "générique" d'IXE-13 dans l'ensemble de la production paralittéraire de l'époque;

- une étude "straussienne" du modèle romanesque d'IXE-13 où se trouve remise en cause une notion importante du structuralisme;

— une étude sociocritique sur l'idéologie du roman policier;

- une étude narratologique à tendance sémiologique sur le modèle du récit, faite par un philosophe. 
Bref, une présentation assez complète dés théories de l'heure où l'originalité de la trouvaille ne craint pas de remettre en cause, plutôt que de les appliquer servilement, certaines notions fondamentales de ces méthodes.

Signalons que ce numéro portant entièrement sur le corpus québécois, aurait pu, par son sujet paralittéraire, figurer, par croisement, dans la série "autres», où se trouvent déjà deux livraisons consacrées à des questions de paralittérature. Preuve. s'il en était besoin, que cette sériation n'était pas, au point de départ, de la plus grande rigueur.

À l'affiche "numéro d'auteur", je tombe sur un numéro ancien, des débuts de la revue, consacré à Alphonse Daudet. un de ces oubliés des études littéraires. Comme il fallait s'y attendre, la méthode historique est présente mais ne domine pas. Après une étude d'un historien sur le domaine de Daudet en Provence, vient une étude qui considère dans sa dimension historique l'échec au théâtre des pièces de Daudet. Quatre études sur six au total interrogent les textes. l'une par le biais de l'analyse fonctionnelle inspirée de Barthes et Genette. l'autre par la méthode linguistique de l'analyse des idéologies, une troisième par la théorie générique du conte, une dernière enfin par la méthode comparatiste sur la fortune de Daudet en Allemagne.

L'honneur est sauf, on le voit, dès les débuts de la revue et à propos d'un auteur somme toute assez «traditionnel». La diversité, assortie de modernité. est déjà ici une marque. Deux contributions sont de Laval, quatre viennent de l'extérieur du Québec.

Pour la catégorie "autres": cinéma et récit. Sept contributions, dont quatre de Laval, deux d'Europe, une du Québec. Deux sont des analyses d'idéologies inspirées des méthodes marxiennes; deux réflexions théoriques sur la narratologie littéraire appliquée au récit cinématographique; suivent deux analyses sémiologiques fondées sur les théories linguistiques. Enfin, une étude sociologique sur le cinéma québécois. Là aussi, diversité se conjugue avec modernité des approches.

Faute d'une analyse exhaustive de tout le corpus d'Êtudes littéraires en fonction des méthodes employées par les collaborateurs. cet échantillonnage nous enseigne, s'il en était encore beșoin, que la revue se situe d'emblée dans l'actualité des méthodes, assouplie par une très grande diversité des approches au sein même de chaque numéro. Le caractère visiblement créateur de nombreuses contributions indiquerait plutôt que cette tendance n'est pas l'effet d'une mode mais bien une véritable politique éditoriale.

Si l'on devait, pour conclure, définir Études littéraires, telle que cette revue apparaît au terme de notre survol, nous dirions qu'elle se singularise par trois caractères:

- une interdisciplinarité d'une grande souplesse;

- une collaboration marquée par l'ouverture internationale et interuniversitaire, combinée à un troisième facteur qui est loin d'être négligeable;

- une place plus qu'honorable faite aux étudiants-chercheurs engagés dans des projets collectifs de recherches. 